\title{
Design Colaborativo e Customização em Massa: uma Interseção de Conceitos Através da Revisão Bibliográfica Sistemática
}

\author{
Collaborative Design and Mass Customization: An Intersection of Concepts Through \\ a Systematic Bibliographic Review
}

\author{
FONTANA, Isabela; Doutoranda; Universidade Federal do Paraná \\ isabelafontana@gmail.com \\ HEEMANN, Adriano; Doutor; Universidade Federal do Paraná \\ adriano.heemann@gmail.com
}

\section{Resumo}

Com o intuito de tornar a pesquisa sobre a colaboração no design de produtos para a customização em massa (CM) mais abrangente, o presente artigo insere á uma análise anterior sobre esses conceitos, uma nova análise dos conceitos específicos isolados de design colaborativo e da CM na tentativa de identificar uma possível intersecção entre os dois assuntos e incluindo a literatura consolidada sobre esses conceitos em separado, que não foram identificadas na busca dos termos em conjunto. Para tanto, o procedimento executado foi uma revisão bibliográfica sistemática (RBS), que resultou em um quadro teórico de síntese que classifica a interseção entre os assuntos desta pesquisa. Com esse quadro é possível identificar lacunas e ênfases que as publicações descrevem em suas pesquisas. Isto torna possível listá-las e usá-las como fatores que merecem maior atenção no processo de design de produto para CM.

Palavras Chave: design colaborativo, customização em massa, desenvolvimento de produto e revisão bibliográfica sistemática.

\begin{abstract}
In order to make the research about collaborative product design for mass customization (MC) more comprehensive, this article introduces an earlier analysis of these concepts, the analysis of specific isolated concepts of collaborative design and CM in attempt to identify a possible intersection between the two subjects and including the consolidated literature on these separate concepts that were not identified in the search for the terms together. For this, the procedure performed was a systematic literature review, which resulted in a theoretical framework of synthesis. With this framework it is possible to identify gaps and emphases that the publications describe in their research. This makes it possible to list them and use them as factors that deserve more attention in the product design process for CM.
\end{abstract}

Keywords: collaborative design, mass customization, product development and systematic literature review. 


\section{Introdução}

Customização em massa (CM), termo utilizado pela primeira vez no livro Future Perfect (DAVIS, 1996) em sua primeira edição em 1987, é a junção de dois conceitos: a produção em massa e a customização. Desde os anos 1990 empresas estão personalizando produtos que tradicionalmente fabricam em massa.

Assim, a CM visa satisfazer necessidades individuais dos clientes, tentando manter a eficiência da produção em massa. Com ela, cada cliente pode especificar as características de um produto, o que resulta em uma ampla variedade de produtos manufaturados assim como uma ampla variedade de informações advindas desse processo (JIAO et al., 2000).

Já o conceito de colaboração no processo de desenvolvimento de produtos (PDP) define um esforço recíproco entre pessoas de iguais ou diferentes áreas de conhecimento, separadas fisicamente ou não, com um objetivo comum de encontrar soluções que satisfaçam a todos os interessados. Isso pode acontecer compartilhando informações e responsabilidades, organizando tarefas e recursos, administrando múltiplas perspectivas e criando um entendimento compartilhado em um processo de design. A colaboração visa produzir um produto e/ou serviço consistente e completo através de uma grande variedade de fontes de informações com certo grau de coordenação das várias atividades implementadas. Esse processo dependente da relação entre os atores envolvidos, da confiança entre eles e da dedicação de cada parte (FONTANA, 2012).

Por sua definição, ambos os conceitos deveriam andar juntos pois utilizar-se das informações existentes sobre a colaboração no design no contexto da CM seria de grande valia, considerando o aumento da complexidade do desenvolvimento de um produto que se adeque aos preceitos da CM.

O processo design de produtos voltados para a produção através da manufatura tradicional possui metodologias e ferramentas já consolidadas, assim como estudos que abordam a colaboração entre os atores desse processo. Porém, o processo tradicional de design apresenta limitações para conseguir responder as exigências necessárias para que seja possível implementar processos de CM.

Com o intuito de tornar a pesquisa sobre a colaboração no design de produtos para a customização em massa (CM) mais abrangente, o presente artigo insere a análise de Fontana \& Heemann (2016) a análise dos conceitos específicos isolados de design colaborativo e da CM na tentativa de identificar uma possível interseç̧ão entre os dois assuntos e incluindo a literatura consolidada sobre esses conceitos em separado, que não foram identificadas na busca dos termos em conjunto.

Para tanto, seguiu-se uma ordem de procedimentos que estruturam este artigo que se inicia com a apresentação geral do problema, seguindo pelo detalhamento do método utilizado. $A$ partir dos resultados obtidos, foi feita uma análise que permitiu elaborar considerações finais e possíveis desenvolvimentos futuros desta pesquisa.

\section{Método}

Como mencionado anteriormente, a presente investigação busca continuar a análise e determinar a situação atual da pesquisa em colaboração no design voltado para a CM (FONTANA 
\& HEEMANN, 2016). A motivação para a continuidade desta pesquisa continua emergindo da possível interligação entre os conceitos de colaboração no design e o desenvolvimento de produtos para a CM.

Para tanto, foi executada uma revisão bibliográfica sistemática (RBS) com base no procedimento iterativo de processamento de dados de Conforto et al. (2011): fase 1 de "entrada" (definição do problema, objetivos, fontes primárias, strings de busca, critérios de inclusão, critérios de qualificação, métodos e ferramentas, e cronograma), fase 2 de "processamento" (condução das buscas, análise dos resultados, documentação), e fase 3 de "saída" (alertas, cadastro e arquivo, síntese de resultados, e modelos teóricos).

Os bancos de dados utilizado foram a Web of Science, Scopus e Science Direct. Foi feita a busca em três bancos de dados para aumentar a abrangência de áreas de pesquisa e periódicos internacionais indexados e a busca foi feita em duas etapas, utilizando os conceitos principais desta pesquisa: customização em massa e colaboração no desenvolvimento de produtos.

Essas buscas foram feitas utilizando os termos, em inglês, "mass customization", e sua variação "mass customisation"; "product development", juntamente com o termo "collaboration". Esta busca teve como objetivo identificar publicações que englobam como tópicos processos colaborativos de desenvolvimento de produtos para a $\mathrm{CM}$, indicando o intervalo de tempo do ano seguinte á análise inicial de 2016 (FONTANA \& HEEMANN, 2016). Esta busca resultou em 36 artigos estão disponíveis na forma de artigo completo digital nas bases de dados utilizada e alinhados com o tema desta pesquisa.

Seguiu-se então para a análise de conteúdo dos artigos completos resultantes para a identificação de características específicas dos assuntos e processos que abordam. 0 escopo estipulado de conceitos para que esses artigos fossem mantidos como pertinentes para a presente pesquisa: trabalhos que tratem de processos de design de produtos ou serviços para a CM e que utilizem algum tipo de colaboração entre os participantes desse processo.

A análise dos artigos resultou em uma síntese e estruturação de dados que permitiu identificar quais são as principais ênfases nas pesquisas atuais encontradas, quais as lacunas que este grupo de artigos possui quanto ao design colaborativo para a CM e suas interseções.

A RBS adotada proporcionou resultados, que são detalhados na próxima seção e que permitem uma análise que precede sua síntese e estruturação da situação atual da pesquisa sobre colaboração no design voltado para a CM.

\section{Resultados}

A RBS resultou em uma lista com 39 publicações que, dentre essas, 23 vieram da busca sobre $\mathrm{CM}$ e 13 da busca sobre desenvolvimento de produtos e colaboração.

\subsection{Customização em Massa}

Iniciando a análise temos o artigo de Zhou et al. (2017) propõem entender a experiência do usuário a partir de uma perspectiva de decisão de escolha em relação à percepção dos usuários de perfis de projeto alternativos de um produto. Visam com isso demonstrar que é de vital importância combinar fator afetivo com fator cognitivo durante a tomada de decisão sobre o design do produto. Geralmente adota-se modelos de decisão normativa sob o pressuposto de que 
a tomada de decisão humana é baseada em uma análise de custo-benefício deliberada, sendo que o design da experiência do usuário tornou-se um fator importante do sucesso do produto (Zhou et al., 2017).

Já segundo Zheng, et al. (2017c) as empresas têm se esforçado para fornecer produtos altamente customizados para satisfazer os requisitos individuais de seus clientes, de forma econômica. Para alcançar o design centrado no cliente, tanto as tecnologias de informação (por exemplo, Web 2.0, internet das coisas e realidade virtual) e técnicas de fabricação (por exemplo, design adaptável, sistema de produção reconfigurável e manufatura aditiva) são consideradas as ferramentas habilitadoras (Zheng, et al., 2017c).

Zheng, et al. (2017b) aborda a manufatura aditiva (MA) ou a impressão em 3D, como uma tecnologia habilitadora para customização ou customização em massa. Essa tecnologia foi desenvolvida rapidamente nos últimos anos. Várias ferramentas de design, materiais, máquinas e agências de serviços podem ser encontradas no mercado utilizando essa tecnologia. As escolhas são abundantes, mas os usuários podem ser facilmente confundidos quanto ao processo de MA que devem usar (Zheng, et al., 2017b).

Zheng et al. (2017a) falam dos nichos de mercado das empresas chamadas de one-of-akind (OKP) dependem principalmente dos produtos altamente customizados e com eficiência de massa. Para conseguir isso, capturar e analisar a voz do cliente de forma rápida e precisa já no início da fase de design do produto desempenha um papel importante. No entanto, devido aos seus recursos limitados e baixo orçamento, as atuais empresas da OKP, que hoje em dia dispõem seus produtos na internet, podem não identificar e analisar os requisitos do cliente com precisão e eficácia, nem sequer pagar a despesa de um processo de design do produto mais complexo. É uma necessidade para eles superar essas falhas adotando métodos inovadores de forma efetiva (Zheng et al., 2017a)

Zhang, M. et al. (2017) discorre sobre como a modularidade do produto também motiva a empresa a se comunicar com os seus fornecedores sobre considerações de qualidade e mudanças de design e envolver fornecedores no design e melhoria de produtos porque os módulos devem ser combinados com outros componentes que não necessariamente vêm do mesmo fornecedor. $A$ modularidade do produto permite que os participantes do processo de design cooperem para resolver problemas de conflito e qualidade padronizando as interfaces entre módulos, melhorando a integração de qualidade interna. Por isso, também aumenta a integração da qualidade do fornecedor indiretamente através da integração de qualidade interna (Zhang, M. et al., 2017).

A otimização da produção combinando impressão 3D e fabricação tradicional é discutida a partir de um ponto de vista quantitativo no artigo de Zhang, L et al. (2017). Um modelo matemático de otimização de fabricação considerando o tamanho do lote é proposto e um algoritmo de evolução diferencial (é introduzido para otimizar o modelo. Os testes numéricos sobre o modelo e o algoritmo revelam as mudanças quantitativas com a introdução da impressão em 3D. O modelo pode ser aplicado para otimizar a fabricação considerando o tamanho do lote que combina impressão 3D e fabricação tradicional. (Zhang, J.-L. et al., 2017)

As técnicas de manufatura aditiva (MA) são ideais para produzir produtos customizados devido à sua alta flexibilidade de design. Apesar dos estudos anteriores sobre produtos customizados produzidos especificamente pela MA, como implantes biomédicos e próteses, a 
otimização simultânea de componentes, materiais, processos de MA e parâmetros dimensionais, continua sendo um desafio (Yao et al., 2017).

Wikner et al., (2017) discorre considerando a perspectiva da cadeia de suprimento para $\mathrm{CM}$, afirmando que dada a necessidade das cadeias de suprimentos modernos de sobreviver e prosperar em ambientes turbulentos e voláteis causados pelo ciclo de vida do produto reduzido, maior demanda por produtos e serviços customizados e mudanças constantes no mercado, a agilidade tornou-se uma capacidade chave para ser alcançada.

Já Wei et al. (2017) explica que o design de famílias de produtos foi reconhecido como um meio eficiente e efetivo para ofertar uma variedade de produtos suficientes para satisfazer uma gama de consumidores e dar suporte à CM. Como o método mais efetivo para satisfazer o crescente desejo do individual, a tecnologia baseada no design de plataforma de produtos foi um tema muito abordado nos últimos anos (Wei et al., 2017).

Mas o lado negativo do design de produtos baseados em plataforma foi exposto gradualmente também nos últimos anos, devido aos produtos, membros de uma família, compartilham muitos elementos comuns para destacar a individualidade. Por esse motivo, a pesquisa sobre design de família de produtos flexíveis é considerada importante por Wei et al. (2017). Comparada a plataforma do produto tradicional, a plataforma do produto flexível considera os fatores de incerteza para o design do produto no futuro. A plataforma flexível baseada na incerteza dos requisitos dinâmicos é o método mais efetivo para enfrentar o problema da CM (Wei et al., 2017).

Wang et al. (2017) explica que no atual mercado global competitivo e na economia baseada no conhecimento, os requisitos do usuário se tornam uma importante informação de entrada para as empresas desenvolverem novos produtos e um fator crítico para impulsionar a evolução do design colaborativo de produtos. Enquanto isso, não há consenso sobre a melhor forma de apoiar atividades de conhecimento e desafios significativos para o gerenciamento de informações de design que permeiam o desenvolvimento rápido de produtos colaborativos considerando a exigência dinâmica do usuário (Wang et al., 2017).

A configuração do produto, uma tecnologia amplamente utilizada no design de famílias de produtos, é uma das tecnologias mais eficazes para estratégias de CM, que vêm sido implementadas por muitas empresas durante os últimos anos (Wang, L. et al., 2017). No entanto, a CM precisa englobar o gerenciamento de todo o ciclo deste produto customizado. A fim de auxiliar o desenvolvimento da $\mathrm{CM}$, é essencial ampliar a tecnologia de configuração para o processo de planejamento da família de produtos, que é a essência tecnológica do processo de configuração de produtos (Wang, L. et al., 2017).

Vogel-Heuser et al., (2017) afirmam em seu artigo que as tendências modernas de fabricação de produtos são definidas pela $\mathrm{CM}$, onde são mantidos lotes pequenos e alta variabilidade de tipos de produtos.

Vanderroost et al., (2017) falam que a CM se enquadra no contexto da fabricação inteligente e da quarta revolução industrial, que está atualmente em andamento (mais conhecida como Indústria 4.0) que visa interligar continuamente todas as etapas (pesquisa, design, teste, produção e controle de qualidade) e sistemas de um processo de fabricação.

Ülkü e Hsuan (2017) afirmam que a modularidade é um forte conceito e prática no 
desenvolvimento de produtos sustentáveis e, portanto, na produção, o que, por sua vez, pode aumentar o consumo sustentável. As descobertas do estudo descrito neste artigo têm implicações diretas para o gerenciamento reverso da cadeia de suprimentos, e as empresas devem levar essas descobertas no início da fase de design do produto (Ülkü e Hsuan, 2017).

Ilidis e Kleiza (2017) afirmam em seu artigo que empresas que ofertam produtos voltados para CM encontram os seguintes problemas: estimativa de capacidade incorreta, aceleração desnecessária, estrangulamentos e outros erros de planejamento e produção.

Já Telfer et al. (2017) falam sobre a integração da otimização virtual no processo de design para palmilhas demonstrou proporcionar proteção do tecido plantar aprimorada para indivíduos em risco de ulceração plantar. O uso de simulações virtuais utilizando técnicas de modelagem numérica oferece uma abordagem potencial para otimizar esses dispositivos (Telfer et al., 2017).

A customização é um paradigma de fabricação emergente para atender às necessidades diversificadas do cliente. O estudo de Tan et al. (2017) propõe uma estrutura para produzir produtos customizados de forma eficiente. Uma abordagem para a melhor combinação de diferentes tipos de módulos é proposta para construir uma arquitetura de montagem adequada. $\mathrm{A}$ modelagem baseada em esboço, que facilita a criação e modificação fáceis de modelos pelos clientes, é apresentada como uma chave para o design deste tipo de produto. Um sistema cibernético fornece a plataforma para o design colaborativo e a co-criação de dos produtos (Tan et al., 2017).

Sun et al., (2017) abordam sobre como a modularização desempenha um papel fundamental no desenvolvimento do sistemas produto-serviço (PSS) para abordar e apoiar o design conceitual individual. Os requisitos funcionais do PSS podem ser identificados e depois classificados em diferentes grupos usando um algoritmo de agrupamento. A estrutura do PSS é composta por uma série de diferentes tipos de módulos físicos e módulos de serviço, onde é necessário identificar a partição de módulo adequada para facilitar o design customizado do PSS (Sun et al., 2017).

Muitos fabricantes hoje estão se esforçando para oferecer sistema de produto-serviço (PSS) de alto valor agregado devido ao aumento da concorrência e da pressão ambiental. As atividades de design de PSS enfrentam uma variedade de desafios, como um alto nível de customização, bem como os desafios resultantes, ou seja, requisitos ocultos na fase de uso do produto, potenciais conflitos de atributos de design e complexidade interna dos processos de serviço (Song e Sakao, 2017).

No entanto, as ideias existentes para a customização do PSS são fragmentadas e insuficientes para ajudar os fabricantes. Assim, é necessário desenvolver um suporte sistemático e abrangente para resolver esses problemas. Para apoiar a customização do PSS nas fases iniciais do design, o estudo de Song \& Sakao (2017) propõe uma estrutura de projeto que envolve um processo de design. A estrutura de projeto proposta é baseada em módulos e, portanto, flexível de acordo com as necessidades do usuário. Além disso, tira proveito de alguns métodos já existentes no design (Song e Sakao, 2017).

Schonberger e Brown (2017) afirmam que a produção concorrente (PC) implica equipar as fábricas com múltiplas unidades produtivas para produzir múltiplos pedidos de clientes simultaneamente. Os principais objetivos da PC são reduzir os prazos de entrega para os clientes e 
os estoques de produtos. Para a empresa, benefícios de longo prazo como um todo incluem melhor retenção de clientes, penetração no mercado e crescimento de vendas. Numa época em que os clientes exigem cada vez maior variedade dos fabricantes, a PC é oportuna, possibilitando a colheita dos benefícios da capacidade de resposta, mantendo os custos de produção baixos o suficiente para a competitividade (Schonberger e Brown, 2017).

Vários fatores foram identificados afetando as intenções para a CM de um produto e do próprio processo. Ribeiro et al. (2017) afirma que a intenção de comprar produtos de vestuário customizados em massa online é diretamente influenciada pelas características situacionais: desejo de produtos exclusivos, necessidade de toque, necessidade de simplicidade, necessidade de realidade e envolvimento no vestuário.

Por fim, Relich e Pawlewski (2017) afirmam que uma das tendências contemporâneas da economia global é a CM, que se baseia em modificações de produtos existentes em vez de projetar tudo de novo. O avanço da tecnologia da informação ajuda as empresas atuais a gerenciar processos de negócios e coletar dados que podem ser uma fonte potencial de informações. As especificações de produtos anteriores fornecem informações de design, custo e tempo de projetos passados que podem ser a base para o design de novos produtos (Relich e Pawlewski, 2017).

\subsection{Colaboração no design}

No que tange a colaboração no processo de design de produtos, publicações sobre o assunto abordam os mais diversos processos colaborativos assim como participantes da colaboração.

Para Zhang et al. (2017) em uma economia de mercado turbulenta, o papel dos fornecedores no design de novos produtos do fabricante recebeu grande atenção das empresas e dos pesquisadores. Foram apresentadas por Zhang et al. (2017) evidências empíricas substanciais sobre a contribuição dos fornecedores para enfrentar os desafios de um menor ciclo de vida do produto, de uma resposta mais imediata e fluxos de informação mais rápidos.

A velocidade que uma empresa consegue colocar um novo produto no mercado desde o inicia do seu desenvolvimento é extremamente importante no mercado de hoje, que se caracteriza por um ciclo de vida curto do produto, uma resposta rápida e fluxos de informação rápidos. Essa velocidade influencia significativamente a competitividade a longo prazo das empresas. O motivo dessa condição é a realidade de que as empresas, que introduzem novos produtos mais rapidamente do que seus concorrentes, podem desfrutar de mais vantagens, como manter a liderança da tecnologia e manter um relacionamento mais próximo com os clientes. No entanto, melhorar essa velocidade do design e entrega de um novo produto envolve inúmeros desafios devido à crescente complexidade do produto e ao ambiente econômico turbulento (Zhang, Y. et al., 2017).

Yan e Azadegan, (2017) explicando que o design inter-organizacional de novos produtos também é arriscado. É mais desafiador incorporar o conhecimento externo à medida que o conhecimento dos participantes externos se torna mais distante do da empresa principal. A coordenação de tarefas interdependentes de um projeto e a resolução de conflitos relacionais com diversas pessoas de fora da organização são onerosas, o que pode levar a necessidade de 
atenção gerencial longe do design básico. Esses desafios são particularmente salientes quando o objetivo de um projeto de design inter-organizacional é desenvolver produtos altamente inovadores com bons retornos financeiros. Assim, é importante entender como as estratégias design inter-organizacionais influenciam a novidade do produto e o desempenho financeiro do produto (Yan e Azadegan, 2017).

Atualmente existe também uma tendência para que as empresas parem de ofertar produtos e passem ofertar soluções para atender melhor as necessidades de seus clientes e reduzir o impacto ambiental. Esse desenvolvimento baseado na solução de um problema e não no produto tem uma integração associada de dispositivos inteligentes que contribui para aumentar a complexidade desse tipo de sistema. A capacidade de desenvolver processos, métodos e ferramentas para lidar com esse tipo de oferta é um fator crítico para as empresas de hoje (WIESNER et al., 2017). Ainda assim, em muitos casos, é difícil encontrar pessoas adequadamente treinadas e ferramentas de desenvolvimento suficientemente integradas para soluções complexas, especialmente no caso das pequenas e médias empresas (WIESNER et al., 2017).

No mercado global competitivo de hoje e na economia baseada no conhecimento, o requisito do usuário torna-se uma importante informação de entrada para as empresas desenvolverem novos produtos e um fator crítico para impulsionar a evolução do design colaborativo de produtos (Wang et al., 2017). Enquanto isso, não há consenso sobre a melhor forma de apoiar atividades de conhecimento e desafios significativos para o estabelecimento de gerenciamento de informações de design para o desenvolvimento de produtos colaborativos rápidos com os requisitos dinâmicos do usuário (Wang et al., 2017).

O estudo de Trott e Simms (2017) aprofunda o conhecimento de rotinas e atividades organizacionais no processo de inovação das indústrias de baixa e média tecnologia. A análise empírica baseia-se em quatro estudos de caso de inovação de novos produtos, extraídos de uma seção transversal do setor de alimentos embalados. Essas descobertas sustentam a visão de que as indústrias de baixa e média tecnologia dependem de atividades não-formais de pesquisa e desenvolvimento (P\&D), como interação firme e experiências compartilhadas (Trott e Simms, 2017).

Já o estudo de Suh et al. (2017) propõe aumentar a utilização simultânea de mensurações no design do produto, mapeando os possíveis e potenciais usos de uma mensuração para acadêmicos e profissionais. Uma solução para melhorar a usabilidade da mensuração no gerenciamento de produtos, mostrando que uma mensuração pode ser desenvolvida usando uma metodologia pluralista para que os resultados possam ser incorporados nas atividades de design dos profissionais e quando a distância entre teoria e a prática é um problema de produção de conhecimento (Suh et al., 2017).

Story el al. (2017) aborda o processo de servitização de produtos que envolve fabricantes que desenvolvem ofertas de serviços para aumentar a receita e o lucro. Serviços avançados, em particular, podem facilitar uma organização mais orientada para o serviço e afetar significativamente os processos de negócios dos clientes. No entanto, as abordagens de servitização são muitas vezes discutidas exclusivamente do ponto de vista do fabricante; negligenciando o papel de outros atores participantes do processo. Adotando uma perspectiva multi-ator, o estudo de (Story et al., 2017) investiga as perspectivas do fabricante, do 
intermediário e do cliente para identificar recursos complementares e competitivos dentro da rede do fabricante, exigido para serviços avançados.

Para Steimer et al. (2017) as empresas são desafiadas a iniciar o planejamento do sistema de fabricação em uma fase anterior. Para enfrentar esse desafio, uma abordagem é a utilização de informações das primeiras fases de desenvolvimento de produto. Isso requer uma base de informações consistente para todos os processos que não estão disponíveis até agora. Para alcançar esta base de informações, dentro do design, novos métodos, como a engenharia de sistemas baseados em modelo são usados. Este é um método para projetar sistemas complexos usando um modelo de sistema central que permite um fácil intercâmbio de dados entre todas as disciplinas de design (Steimer et al., 2017).

Stefan e Bengtsson (2017) discorrem que há até hoje uma abundante evidência sobre a maneira como as relações de abertura de desempenho são moldadas, mas fluxos paralelos de pesquisa apontam para uma intrincada relação entre apropriabilidade e abertura. Consequentemente, enquanto a abertura pode revelar amplas oportunidades, os riscos da apropriação indevida também devem ser contabilizados em processos abertos de inovação, pois podem afetar o desempenho. Este artigo visa investigar os efeitos de três grupos de mecanismos de proteção de propriedade intelectual (formal, semiformal e informal) e abertura (em termos de profundidade de colaboração com oito tipos de parceiros) em dois tipos de desempenho de inovação (eficiência e novidade) em fases de inovação (Stefan e Bengtsson, 2017).

Spring et al. (2017) olham a colaboração da perspectiva das intervenções políticas dos governos. Afirmam que as intervenções políticas dos governos para alterar a estrutura da atividade econômica foram descartadas ou ignoradas pelos especialistas em gerenciamento de operações. No entanto, nos últimos anos, essas medidas de "política industrial" ganharam apoio crescente nas economias desenvolvidas, particularmente em relação à fabricação. Este artigo argumenta que a fabricação contemporânea em economias de alto custo está enraizada na inovação tecnológica. Como tal, pode ser reforçada por intervenções de política industrial que impedem falhas de sistemas no processo de transformar a inovação tecnológica em produtos comercialmente viáveis (Spring et al., 2017).

Si et al. (2017) também discorre sobre sistemas produto serviço (PSS). Explicam que cada vez mais, as organizações de fabricação competem ao desenvolver PSS em vez de oferecer produtos sozinhos. Para transformar-se em prestadores de serviços avançados, no entanto, as empresas de fabricação centradas no produto precisam superar uma série de barreiras. Enquanto estudos anteriores destacaram o potencial de ensino/aprendizagem de "gamificação" (o uso de ideias e técnicas encontradas no jogo), as oportunidades para aproveitar essa abordagem para ajudar a enfrentar essas barreiras ainda não foram totalmente realizadas (Shi et al., 2017).

O ambiente virtual está facilitando a busca pela inovação. Por sua vez, as empresas são capazes de converter ideias de clientes em produtos ou serviços, melhorando seus retornos financeiros (Scuotto et al., 2017). Os clientes se tornam os novos pioneiros. Assim, ao adotar uma perspectiva de inovação liderada por clientes, este trabalho busca medir o efeito sobre o retorno sobre o investimento do uso de redes sociais como drivers externos para suportar processos internos de busca de inovação (Scuotto et al., 2017).

Por fim, Schuh et al. (2017) explicam que atualmente, os fabricantes de ferramentas muitas vezes ainda são pouco integrados nos processos dos clientes, o que complica 
significativamente a redução dos tempos de desenvolvimento. Para melhorar a integração, os processos e serviços de alto valor agregado devem ser oferecidos aos seus clientes para participar dos processos de desenvolvimento de produtos de todos os clientes. Devido ao fato de que os clientes ainda exigem qualidade de ferramenta constantemente alta, são necessários novos procedimentos e tecnologias para alcançar a maturidade precoce de um produto (Schuh et al., 2017).

Para que seja possível identificar se os resultados enumerados e descritos permitem a identificação das principais ênfases ou possíveis lacunas no que tange a colaboração no design para a CM, uma síntese estruturada foi efetuada. Esta síntese é descrita a seguir, indicando como os resultados foram analisados para alcançar o objetivo geral desta pesquisa.

\section{Discussão}

Com base na classificação de participantes da colaboração em processos de CM de Fontana \& Heemann (2016) e nos conceitos base esperados nos artigos, os autores analisados foram sintetizados e estruturados para uma melhor visualização das lacunas, ênfases e interseções nas pesquisas sobre esses conceitos, considerando o contexto da colaboração no design para a CM.

Os quadros foram preenchidos considerando uma escala de 0 a 2 onde: 0 indica que o conceito indicado na primeira linha não está presente no artigo; 1 indica que o conceito é comentado no artigo e 2 o conceito está presente e é discutido no artigo.

O somatório de cada conceito aparece na última linha do quadro, classificando a profundidade com que os conceitos foram abordados nos artigos, através das cores verde (o conceito já foi bem estudado em profundidade), amarelo (o conceito já foi abordado, mas não em profundidade), e vermelho (o conceito foi pouco abordado das pesquisas).

\subsection{Customização em Massa (CM)}

A análise sobre os artigos sobre CM (Figura 1) demonstrou que a sua grande maioria aborda de forma consistente os conceitos de design de produto, customização, processos colaborativos com os clientes e habilitadores para a produção de produtos customizados em massa.

Já a colaboração com designers, com fabricantes e com os gestores das organizações aparecem como conceitos presentes das pesquisas, porém sem serem abordados de forma concisa.

Por fim, a colaboração com fornecedores, a colaboração com pesquisadores acadêmicos, a colaboração com engenheiros, fatores que podem ser considerados como críticos para a $\mathrm{CM}$ e habilitadores para a colaboração entre os participantes aparecem como conceitos pouco abordados nas pesquisas.

Os conceitos que se mostraram como abordados, mas não em profundidade (amarelo), e pouco abordados nas pesquisas (vermelho) podem ser considerados lacunas importantes na pesquisa atual sobre a CM no contexto da colaboração no design. 
Figura 1 - Quadro de síntese das pesquisas publicadas em 2017 sobre customização em massa

RELICH, M.; PAWLEWSKI, P.

RIBEIRO, L. S.; DUARTE, P. A. O.; MIGUEL, R.

SCHOENWITZ, M. et al.

SCHONBERGER, R. J.; BROWN, K. A.

SKELLERN, K.; MARKEY, R.; THORNTHWAITE, L.

SONG, W.; SAKAO, T.

SUN, J. et al.

TAN, C. et al.

TELFER, $S$. et al.

TILINDIS, J.; KLEIZA, V.

ÜLKÖ, M. A.; HSUAN, J.

VANDERROOST, M. et al.

VOGEL-HEUSER, B. et al.

WANG, L.; ZHONG, S. S.; ZHANG, Y. J.

WANG, Y.; YU, S.; XU, T.

WEI, W. et al.

WIKNER, J. et al.

YAO, X.; MOON, S. K.; BI, G.

ZHANG, J.-L; ZHANG, Z; HAN, Y.

ZHANG, M. et al.

ZHENG, P. et al. A

ZHENG, P. et al. B

ZHENG, P. et al. C

ZHOU, F. et al.

\begin{tabular}{|c|c|c|c|c|c|c|c|c|c|c|c|c|}
\hline 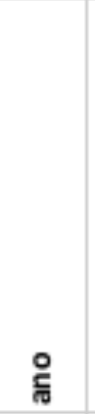 & 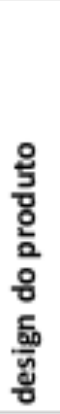 & 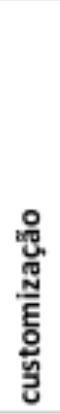 & 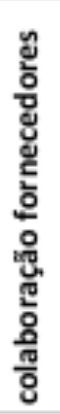 & 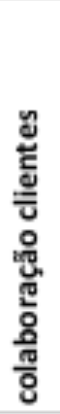 & 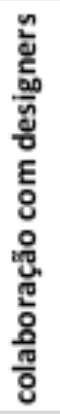 & 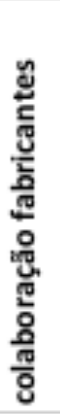 & 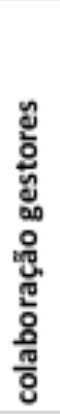 & 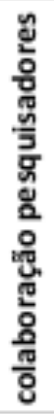 & 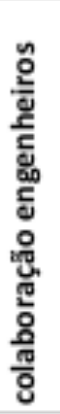 & 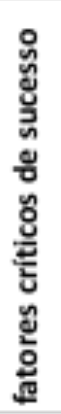 & 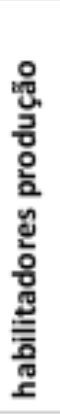 & 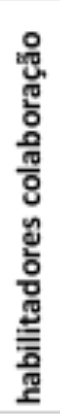 \\
\hline 2017 & 2 & 2 & 1 & 1 & 1 & 1 & 1 & 0 & 1 & 2 & 2 & 2 \\
\hline 2017 & 2 & 2 & 0 & 2 & 0 & 0 & 0 & 0 & 0 & 2 & 1 & 1 \\
\hline 2017 & 1 & 1 & 0 & 0 & 0 & 0 & 0 & 0 & 0 & 0 & 1 & 0 \\
\hline 2017 & 1 & 2 & 1 & 0 & 0 & 1 & 1 & 0 & 1 & 0 & 2 & 0 \\
\hline 2017 & 1 & 1 & 1 & 1 & 1 & 1 & 1 & 1 & 1 & 0 & 0 & 0 \\
\hline 2017 & 2 & 2 & 0 & 2 & 2 & 2 & 0 & 0 & 0 & 1 & 2 & 1 \\
\hline 2017 & 2 & 2 & 2 & 2 & 2 & 2 & 2 & 0 & 1 & 0 & 2 & 1 \\
\hline 2017 & 2 & 2 & 0 & 2 & 1 & 1 & 1 & 0 & 1 & 1 & 2 & 2 \\
\hline 2017 & 2 & 2 & 0 & 0 & 0 & 0 & 0 & 0 & 0 & 0 & 2 & 1 \\
\hline 2017 & 0 & 2 & 1 & 1 & 0 & 2 & 2 & 0 & 0 & 1 & 0 & 0 \\
\hline 2017 & 2 & 2 & 2 & 2 & 2 & 2 & 2 & 0 & 2 & 1 & 2 & 0 \\
\hline 2017 & 2 & 2 & 2 & 2 & 2 & 2 & 2 & 2 & 2 & 2 & 2 & 1 \\
\hline 2017 & 1 & 1 & 0 & 0 & 0 & 0 & 0 & 0 & 0 & 0 & 2 & 0 \\
\hline 2017 & 2 & 2 & 2 & 2 & 2 & 2 & 2 & 0 & 2 & 1 & 2 & 0 \\
\hline 2017 & 2 & 2 & 0 & 2 & 2 & 2 & 2 & 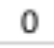 & 2 & 1 & 2 & 0 \\
\hline 2017 & 2 & 2 & 1 & 1 & 2 & 1 & 1 & 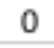 & 0 & 1 & 2 & 0 \\
\hline 2017 & 0 & 2 & 2 & 2 & 0 & 2 & 2 & 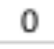 & 0 & 1 & 2 & 0 \\
\hline 2017 & 2 & 2 & 0 & 0 & 0 & 0 & 0 & 0 & 0 & 0 & 2 & 0 \\
\hline 2017 & 2 & 2 & 0 & 0 & 0 & 0 & 0 & 0 & 0 & 0 & 2 & 0 \\
\hline 2017 & 2 & 2 & 2 & 2 & 2 & 2 & 2 & 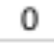 & 2 & 0 & 0 & 0 \\
\hline 2017 & 2 & 2 & 0 & 2 & 2 & 1 & 1 & 0 & 0 & 1 & 0 & 2 \\
\hline 2017 & 1 & 2 & 0 & 1 & 0 & 0 & 0 & 0 & 0 & 0 & 2 & 2 \\
\hline 2017 & 2 & 2 & 0 & 2 & 1 & 2 & 0 & 0 & 0 & 2 & 2 & 2 \\
\hline \multirow[t]{2}{*}{2017} & 2 & 2 & 1 & 2 & 2 & 0 & 0 & 0 & 0 & 1 & 0 & 2 \\
\hline & 39 & 45 & 18 & 31 & 24 & 26 & 22 & 3 & 15 & 18 & 36 & 17 \\
\hline
\end{tabular}

Fonte: os autores

\subsection{Colaboração no design}

A análise sobre os artigos sobre colaboração no design de produtos (Figura 2) demostrou, também, que a sua grande maioria aborda de forma consistente os conceitos de design de produto, processos colaborativos com os gestores e habilitadores para a colaboração entre os participantes do processo de desenvolvimento de produto. 


\begin{tabular}{|c|c|c|c|c|c|c|c|c|c|c|c|c|c|}
\hline & 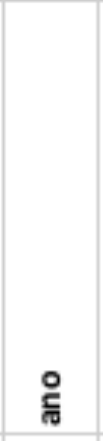 & 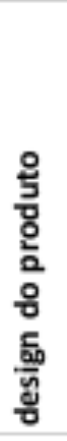 & 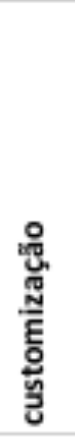 & 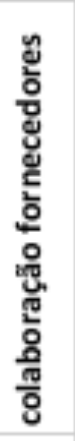 & 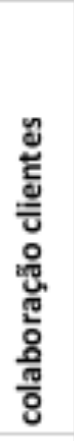 & 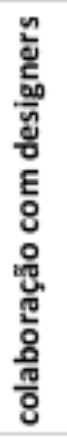 & 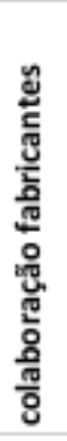 & 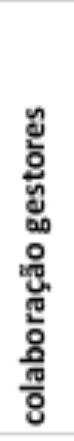 & 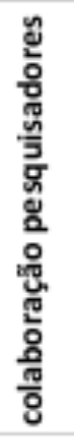 & 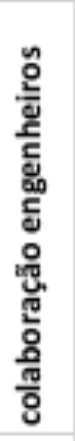 & 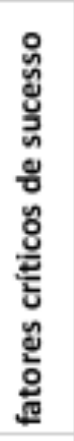 & 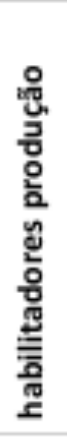 & 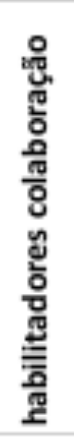 \\
\hline SCHUH, G. et al. & 2017 & 2 & 2 & 1 & 2 & 1 & 2 & 1 & 0 & 1 & 2 & 2 & 2 \\
\hline scuotTo, v. et al. & 2017 & 2 & 0 & 1 & 2 & 1 & 1 & 1 & 2 & 1 & 1 & 0 & 2 \\
\hline SHI, V. G. et al. & 2017 & 2 & 0 & 0 & 0 & 0 & 0 & 0 & 0 & 0 & 2 & 1 & 2 \\
\hline SPRING, M. et al. & 2017 & 1 & 0 & 0 & 0 & 0 & 0 & 2 & 2 & 0 & 1 & 0 & 2 \\
\hline STEFAN, I.; BENGTSSON, L. & 2017 & 2 & 0 & 1 & 0 & 1 & 1 & 1 & 0 & 1 & 1 & 0 & 2 \\
\hline STEIMER, C.; FISCHER, J.; AURICH, J. C. & 2017 & 2 & 0 & 1 & 1 & 1 & 1 & 1 & 1 & 1 & 1 & 0 & 2 \\
\hline STORY, V. M. et al. & 2017 & 2 & 0 & 2 & 2 & 1 & 1 & 1 & 0 & 1 & 2 & 1 & 1 \\
\hline SUH, T. et al. & 2017 & 2 & 0 & 1 & 1 & 1 & 1 & 1 & 2 & 1 & 2 & 0 & 1 \\
\hline TROTT, P.; SIMMS, C. & 2017 & 2 & 0 & 1 & 1 & 1 & 1 & 1 & 2 & 1 & 0 & 0 & 2 \\
\hline WANG, Y.; YU, S.; XU, T. & 2017 & 2 & 2 & 0 & 2 & 2 & 2 & 2 & 0 & 2 & 1 & 2 & 0 \\
\hline WIESNER, S.; NILSSON, S.; THOBEN, K.-D. & 2017 & 2 & 0 & 1 & 1 & 1 & 1 & 1 & 0 & 1 & 2 & 2 & 2 \\
\hline YAN, T.; AZADEGAN, A. & 2017 & 2 & 1 & 2 & 0 & 2 & 2 & 2 & 0 & 2 & 1 & 2 & 2 \\
\hline \multirow[t]{2}{*}{ ZHANG, Y.; WANG, L.; GAO, J. } & 2017 & 2 & 0 & 2 & 0 & 2 & 2 & 2 & 1 & 1 & 0 & 0 & 1 \\
\hline & & 25 & 5 & 13 & 12 & 14 & 15 & 16 & 10 & 13 & 16 & 10 & 21 \\
\hline
\end{tabular}

Fonte: os autores

Indo na direção contrária, os conceitos que estão presentes nos artigos analisados, porém não são abordados em profundidade estão a colaboração com fornecedores, com clientes, com designers, com fabricantes e com engenheiros.

O conceito de customização, a colaboração com pesquisadores acadêmicos e habilitadores para a produção de produtos voltados para a CM aparecem como conceitos não abordados de forma mais aprofundada nas pesquisas.

Os conceitos classificados como "amarelos" e "vermelhos" podem ser entendidos como grandes lacunas na pesquisa atual sobre a colaboração no design de produtos.

É importante ressaltar que nessa busca de termos separados, um único artigo apareceu nas duas buscas (na de CM e na de colaboração no design), o artigo de Wang, Y. et al. (2017), o que indica que esse artigo aparece em uma busca dos termos em conjunto também. Como é de se esperar, por sua característica de aparecer nas duas buscas, este é um dos artigos que mais abordam todos os conceitos indicados no quadro.

\section{Conclusão}

Partindo do objetivo de dar continuidade na identificação da situação atual da pesquisa relacionada ao design colaborativo voltado para a $\mathrm{CM}$, o artigo seguiu etapas que permitiram a identificação das principais ênfases e lacunas dos artigos analisados nesta pesquisa considerando 
a interseção dos dois assuntos.

Após a síntese, pode-se observar pela classificação de cores dada, que existem conceitos que são mais abordados tanto na literatura sobre CM como na literatura sobre colaboração no desenvolvimento de produtos. Os conceitos com menor classificação devem ser considerados como prioridade para pesquisas de aprofundamento sobre colaboração no design para a CM

Sugere-se, para desenvolvimento futuro, identificar nestas pesquisas fatores críticos de sucesso para a colaboração no design para CM a fim de desenvolver possíveis formas, sejam ferramentas, métodos, etc., de lidar com as lacunas encontradas a fim de facilitar o design colaborativo de produtos especificamente voltados $\mathrm{CM}$.

\section{Referências}

CONFORTO, E. C.; AMARAL, D. C.; SILVA, S. L. Roteiro para revisão bibliográfica sistemática: aplicação no desenvolvimento de produtos e gerenciamento de projetos. 8 o Congresso Brasileiro de Gestão de Desenvolvimento de Produto (CBGDP 2011). Porto Alegre, RS - Brasil, 2011.

\section{DAVIS, S. Future Perfect. 1996}

FONTANA, I. M. Fatores críticos de sucesso para a colaboração no design de sistemas produtoserviço. Dissertação (Mestrado em Design), Programa de Pós-Graduação em Design, Universidade Federal do Paraná, Curitiba, 2012.

FONTANA, I. M.; HEEMANN, A. Colaboração no design para a customização em massa: situação e perspectivas. In: Congresso Brasileiro de Pesquisa e Desenvolvimento em Design, 2016, Belo Horizonte. Anais do Congresso Brasileiro de Pesquisa e Desenvolvimento em Design, 2016.

JIAO, J. X.; MA, Q. H.; TSENG, M. M. Towards high value-added products and services: mass customization and beyond. Technovation, v. 23, n. 10, p. 809-821, Oct 2003.

RELICH, M.; PAWLEWSKI, P. A case-based reasoning approach to cost estimation of new product development. Neurocomputing, 2017.

RIBEIRO, L. S.; DUARTE, P. A. O.; MIGUEL, R. Online consumer behaviour of mass-customised apparel products: A hierarchy of traits approach. Journal of Fashion Marketing and Management, v. 21, n. 2, p. 158-171, 2017.

SCHOENWITZ, M. et al. Product, process and customer preference alignment in prefabricated house building. International Journal of Production Economics, v. 183, Part A, p. 79-90, 1// 2017.

SCHONBERGER, R. J.; BROWN, K. A. Missing link in competitive manufacturing research and practice: Customer-responsive concurrent production. Journal of Operations Management, v. 4951, p. 83-87, 3// 2017.

SKELLERN, K.; MARKEY, R.; THORNTHWAITE, L. Identifying attributes of sustainable transitions for traditional regional manufacturing industry sectors - A conceptual framework. Journal of Cleaner Production, v. 140, Part 3, p. 1782-1793, 1/1/ 2017.

$\mathrm{SCHUH}, \mathrm{G}$. et al. Highly Iterative Product Development Within The Tool and Die Making Industry. Procedia CIRP, v. 61, p. 576-581, // 2017. 
SCUOTTO, $\mathrm{V}$. et al. The performance implications of leveraging internal innovation through social media networks: An empirical verification of the smart fashion industry. Technological Forecasting and Social Change, v. 120, p. 184-194, 7// 2017.

SHI, V. G. et al. Using gamification to transform the adoption of servitization. Industrial Marketing Management, v. 63, p. 82-91, 5// 2017.

SONG, W.; SAKAO, T. A customization-oriented framework for design of sustainable product/service system. Journal of Cleaner Production, v. 140, Part 3, p. 1672-1685, 1/1/ 2017.

SPRING, $M$. et al. Creating the competitive edge: A new relationship between operations management and industrial policy. Journal of Operations Management, v. 49-51, p. 6-19, 3// 2017.

STEFAN, I.; BENGTSSON, L. Unravelling appropriability mechanisms and openness depth effects on firm performance across stages in the innovation process. Technological Forecasting and Social Change, v. 120, p. 252-260, 7// 2017.

STEIMER, C.; FISCHER, J.; AURICH, J. C. Model-based Design Process for the Early Phases of Manufacturing System Planning using SysML. Procedia CIRP, v. 60, p. 163-168, // 2017.

STORY, V. M. et al. Capabilities for advanced services: A multi-actor perspective. Industrial Marketing Management, v. 60, p. 54-68, 1// 2017.

$\mathrm{SUH}, \mathrm{T}$. et al. Enhancing the simultaneous utilization of measure in product design for academicpractitioner collaboration. Journal of Product and Brand Management, v. 26, n. 3, p. 312-326, 2017.

SUN, J. et al. Modularization of Product Service System Based on Functional Requirement. Procedia CIRP, v. 64, p. 301-305, // 2017. ISSN 2212-8271.

TAN, C. et al. Product personalization enabled by assembly architecture and cyber physical systems. CIRP Annals - Manufacturing Technology, 2017.

TELFER, S. et al. Virtually optimized insoles for offloading the diabetic foot: A randomized crossover study. Journal of Biomechanics, 2017.

TILINDIS, J.; KLEIZA, V. Learning curve parameter estimation beyond traditional statistics. Applied Mathematical Modelling, v. 45, p. 768-783, 5// 2017.

TROTT, P.; SIMMS, C. An examination of product innovation in low- and medium-technology industries: Cases from the UK packaged food sector. Research Policy, v. 46, n. 3, p. 605-623, 4// 2017.

ÜLKÜ, M. A.; HSUAN, J. Towards sustainable consumption and production: Competitive pricing of modular products for green consumers. Journal of Cleaner Production, v. 142, p. 4230-4242, 2017.

VANDERROOST, $M$. et al. The digitization of a food package's life cycle: Existing and emerging computer systems in the pre-logistics phase. Computers in Industry, v. 87, p. 1-14, 5// 2017

VOGEL-HEUSER, B. et al. Modularity and architecture of PLC-based software for automated production Systems: An analysis in industrial companies. Journal of Systems and Software, v. 131, p. 35-62, 9// 2017.

WANG, L et al. Process configuration based on generative constraint satisfaction problem. 2017 
WANG, Y.; YU, S.; XU, T. A user requirement driven framework for collaborative design knowledge management. Advanced Engineering Informatics, v. 33, p. 16-28, 2017.

WEI, W. et al. Product Family Flexible Design Method Based on Dynamic Requirements Uncertainty Analysis. Procedia CIRP, v. 60, p. 332-337, // 2017.

WIESNER, S.; NILSSON, S.; THOBEN, K.-D. Integrating Requirements Engineering for Different Domains in System Development - Lessons Learnt from Industrial SME Cases. Procedia CIRP, v. 64, p. 351-356, // 2017.

WIKNER, J. et al. IOBPCS based models and decoupling thinking. International Journal of Production Economics, 2017.

YAN, T.; AZADEGAN, A. Comparing inter-organizational new product development strategies: Buy or ally; Supply-chain or non-supply-chain partners? International Journal of Production Economics, V. 183, p. 21-38, 2017.

X.; MOON, S. K.; BI, G. Multidisciplinary design optimization to identify additive manufacturing resources in customized product development. Journal of Computational Design and Engineering, $\mathrm{v}$. 4, n. 2, p. 131-142, 4// 2017.

ZHANG, J.-L.; ZHANG, Z.; HAN, Y. Research on manufacturability optimization of discrete products with 3D printing involved and lot-size considered. Journal of Manufacturing Systems, v. 43, Part 1, p. 150-159, 4// 2017.

ZHANG, M. et al. Linking supply chain quality integration with mass customization and product modularity. International Journal of Production Economics, 2017.

ZHANG, Y.; WANG, L.; GAO, J. Supplier collaboration and speed-to-market of new products: the mediating and moderating effects. Journal of Intelligent Manufacturing, v. 28, n. 3, p. 805-818, 2017.

ZHENG, P. et al. Personalized product configuration framework in an adaptable open architecture product platform. Journal of Manufacturing Systems, v. 43, Part 3, p. 422-435, 4// 2017. a

ZHENG, $P$. et al. A weighted rough set based fuzzy axiomatic design approach for the selection of AM processes. International Journal of Advanced Manufacturing Technology, v. 91, n. 5-8, p. 19771990, 2017. b

ZHENG, P. et al. A system framework for OKP product planning in a cloud-based design environment. Robotics and Computer-Integrated Manufacturing, v. 45, p. 73-85, 2017. c

ZHOU, F. et al. Affective parameter shaping in user experience prospect evaluation based on hierarchical Bayesian estimation. Expert Systems with Applications, v. 78, p. 1-15, 7/15/ 2017. 